\section{LA COMUNICACIÓN PÚBLICA DE ÁMBITOS CIENTÍFICOS Y TECNOLÓGICOS EMERGENTES. PROBLEMAS Y RETOS EN EL CASO DE LA NANOTECNOLOGÍA}

\author{
Javier Gómez-Ferri \\ Departamento de Sociología y Antropología Social \\ Universidad de Valencia \\ javier.gomez-ferri@uv.es \\ José Manuel de Cózar Escalante \\ Departamento de Historia y Filosofía de la Ciencia \\ Universidad de La Laguna \\ jcozar@ull.es \\ Ramón Llopis-Goig \\ Departamento de Sociología y Antropología Social \\ Universidad de Valencia \\ ramon.llopis@uv.es
}

Cómo citar este artículo/Citation: Gómez-Ferri, J.; Cózar Escalante, J.M. y Llopis-Goig, R. (2014). "La comunicación pública de ámbitos científicos y tecnológicos emergentes. Problemas y retos en el caso de la nanotecnología". Arbor, 190 (766): a123. doi: http://dx.doi.org/10.3989/ arbor.2014.766n2015

Recibido: 15 julio 2013. Aceptado: 3 febrero 2014.

RESUMEN: Este trabajo se centra en la comprensión y comunicación pública de la nanociencia y la nanotecnología. La preocupación por las actitudes y los niveles de conocimiento público sobre nanociencia y nanotecnología ha llevado en diversos países a estudiar de manera temprana las percepciones sociales sobre ambas. No ha sido ese el caso de España ni de América Latina, donde existe una carencia tanto de estudios de comprensión pública como de actividades de divulgación científica sobre nanotecnología. Este trabajo tiene un doble objetivo. Primero contextualizar y explicar la situación, especialmente, en la sociedad española. Segundo exponer los resultados de un estudio Delphi realizado a expertos en nanotecnología $(n=38)$ que pretende compensar dicha situación. Los resultados consisten en un conjunto ordenado de contenidos básicos sobre nanotecnología que científicos, periodistas, divulgadores y educadores podrían emplear a la hora de informar, así como también en una serie de cautelas a tener presente.

PALABRAS CLAVE: comunicación pública de la ciencia; divulgación de la ciencia; comprensión pública de la ciencia; cultura científica nanotecnología; nanociencia; método Delphi.

\section{PUBLIC COMMUNICATION OF EMERGING SCIENCES AND TECHNOLOGIES. PROBLEMS AND CHALLENGES IN THE CASE OF NANOTECHNOLOGY}

Copyright: (C) 2014 CSIC. Este es un artículo de acceso abierto distribuido bajo los términos de la licencia Creative Commons Attribution-Non Commercial (by-nc) Spain 3.0.

ABSTRACT: This work deals with public understanding of nanoscience and nanotechnology. Concerns about public attitudes towards both, including a lack of knowledge on the part of the general public, have lead scholars in a number of countries to conduct early studies on the social perceptions of these fields. However, this has not been the case in Spain or Latin America, where studies on the popularisation and public understanding of nanotechnology are almost non-existent. This work has a double aim. First, it seeks to contextualise and explain the Spanish situation in this regard. Second, it aims to communicate the results of a Delphi study involving nanotechnology experts $(n=38)$ and designed to fill the above mentioned gap. These results consist of an ordered set of basic content items on nanotechnology that scientists, journalists and educators should bear in mind, as well as some issues to be mindful of with regard to those content items.

KEYWORDS: Public Communication of science; Science popularisation; public understanding of science; scientific culture; nanotechnology; nanoscience; Delphi Method. 


\section{INTRODUCCIÓN}

La nanociencia y la nanotecnología ${ }^{1}$ son un reciente ámbito de innovación científica y técnica del que se aventura tendrá repercusiones económicas y sociales amplias y muy significativas. Esto plantea importantes desafíos en el marco de las relaciones cienciasociedad, y más particularmente en el campo de la comprensión y comunicación pública de la ciencia. Sobre estos aspectos, lo que más fuertemente llama la atención de la nanociencia y nanotecnología es la existencia de una gran invisibilidad social al respecto, a pesar del hecho de estar presentes en productos tan extendidos como los teléfonos móviles, por citar sólo un caso. Por otro lado, también puede ser llamativa alguna reacción esporádica de oposición. En Francia o Suiza han tenido lugar algunas de ellas, pero las más violentas se han dado en México, donde ha llegado a producirse una cadena de atentados contra centros e investigadores en nanociencia y nanotecnología (Phillips, 2012:579). En todo caso, la situación predominante es la existencia de un gran desconocimiento público y una actitud que oscila más entre el optimismo y la indiferencia que entre el miedo y el rechazo. En muchos de los países tecnológicamente más desarrollados, la preocupación tanto por las actitudes como por los niveles de conocimiento científico de la ciudadanía sobre nanotecnología ha llevado a estudiar de manera temprana las percepciones y reacciones sociales, antes incluso de que se hubiera articulado una opinión pública en torno a ella, quedando pospuesto y casi en un segundo plano lo relacionado con la comunicación social de la nanotecnología. Aunque la nanotecnología no tiene un problema de marketing por ahora (Scheufele, 2006:48), sí que se encuentra en un momento crucial, como advierten diversos autores y organizaciones (Hayhurst, Heckl, Maglio, Türk y Bennett, 2007:229; Petersen, Anderson, Allan y Wilkinson, 2009:513). En este sentido, como señalan Simons, Zimmer, Vierboom, Härlen, Hertel y Böl (2009:1569) "las estrategias para comunicar la nanotecnología deben darse antes de que las noticias negativas puedan copar los titulares". Su postergación puede resultar contraproducente dado que, con los actuales niveles de desconocimiento, las actitudes alarmistas pueden calar fácilmente en la ciudadanía.

En ese contexto, la situación en el caso de España y en los países latinoamericanos se caracteriza por un déficit de actividades de divulgación de la nanotecnología, como también por la ausencia de estudios específicos de percepción pública (Tutor y Serena, 2011; Serena y Tutor, 2011:50)². Es un hecho llama- tivo, aunque no extraordinario, que este déficit no se corresponda con el nivel de desarrollo del campo en países como España o Brasil, que son pioneros en la región y que van seguidos de México, Portugal y Argentina. Por ejemplo, España ocupa el undécimo lugar mundial en la producción científica en nanociencia y nanotecnología y Brasil el decimoséptimo (OEI, 2009). Ante esa situación, los objetivos que se propone este trabajo son, en primer lugar, llamar la atención sobre tales carencias; en segundo lugar, ofrecer una explicación de las mismas; y, finalmente, sentar unas bases que contribuyan a paliar el déficit comunicativo detectado. Para ello hemos aplicado el método Delphi, con el que, gracias a la consulta a un conjunto de expertos en nanotecnología pretendemos producir una información básica que facilite a docentes, periodistas, divulgadores y científicos el acercamiento de los contenidos de la nanotecnología a la sociedad. Entendemos que la información proporcionada por los expertos no es la única información necesaria para tal fin, sino que también debe tenerse en cuenta las demandas informativas del público o públicos, así como el punto de vista de comunicadores y divulgadores y de otros expertos del área de las ciencias sociales conocedores de la nanociencia y nanotecnología. Con estas tres aportaciones, creemos que los elementos para diseñar estrategias de comunicación estarían completos, las cuales no tienen que correr a cargo de los investigadores del campo de la nanociencia y la nanotecnología ni tampoco tienen por qué ser ellos necesariamente los que van a realizar las actividades de divulgación, aunque sí que entendemos que su aportación es fundamental a la hora de identificar las cuestiones más básicas para su mejor comprensión.

En las sociedades democráticas adquiere una especial relevancia la existencia de una ciudadanía informada sobre las diferentes esferas de la actividad humana y de la realidad natural y social; la ciencia y la tecnología son dos de ellas (Bodmer et al., 1985:7). Por razones de índole económica, política, cívica y social es importante que los ciudadanos comprendan aspectos básicos de la ciencia y la tecnología, así como sobre su dinámica y funcionamiento (Gregory y Miller, 1998:10). Para ello el periodismo y la divulgación científica, y la enseñanza de las ciencias desempeñan un papel fundamental. Una ciudadanía formada y bien informada representa un beneficio para los propios individuos y un bien para la sociedad en su conjunto.

Sin embargo, una sensación que está muy presente, y que la nanotecnología confirmaría de momento, es la posibilidad de un aumento de la brecha 
cognitiva entre ciudadanos y científicos (Calvo Hernando, 1997:63). La comprensión de la ciencia y de la tecnología por parte de la sociedad sigue siendo una asignatura pendiente en la mayor parte de países. La tarea a realizar es más urgente si cabe en el caso de ciencias y tecnologías emergentes como las que convergen en la nanoescala (biotecnología, nanotecnología, ciencias cognitivas, tecnologías de la información, robótica, etc.). En el actual contexto de institucionalización de las actividades de fomento y difusión de la cultura científica, conocer y mejorar los niveles de conocimiento público sobre la nanotecnología es una tarea pendiente en la sociedad española, y un paso previo para la participación social en la toma de decisiones.

Teniendo presente esta situación, la investigación emprendida ha abordado diversos aspectos relacionados con la comunicación de la nanotecnología, en particular centrados en los mensajes que se transmiten. Primero realizando un análisis y un diagnóstico de las principales dificultades y problemas existentes. Luego realizando una consulta a los expertos, aplicando la metodología Delphi, con el fin de identificar las cuestiones más básicas que el público debería conocer sobre nanotecnología, así como aquellas que pueden ser más complejas o prestarse a ser mal interpretadas, y que periodistas y divulgadores pueden tener también presentes en el ejercicio de su actividad profesional. Como resultado hemos obtenido un conjunto ordenado de contenidos sobre nanotecnología útiles en la divulgación e información sobre la nanotecnología misma. Primero, identificando los contenidos básicos; luego, aquellos que pueden revestir mayores dificultades de comprensión para el público.

\section{MARCO TEÓRICO Y ESTADO DE LA CUESTIÓN}

La nanociencia y la nanotecnología se suelen definir como la investigación, el desarrollo y la innovación científica y técnica que se ocupan del estudio, control y manipulación de la materia a escala nanométrica. Son escalas del orden de la mil millonésima parte de metro. Sus orígenes inmediatos suelen situarse a mediados de la década de 1980 , en concreto una fecha clave es la construcción del primer microscopio de efecto túnel (STM) en 1981 por G. Binning y H. Rohrer, razón por la cual se les concedió el Premio Nobel en 1986. A pesar de ello, la nanociencia y la nanotecnología pasaron bastante desapercibidas hasta la puesta en marcha de la NNI (Nanotechnology National Initiative) en el año 2000 por parte del gobierno estadounidense, presidido en aquel momento por B. Clinton.
La clave de la nanociencia y la nanotecnología reside en que a esas escalas la materia tiene propiedades diferentes de las que presenta a escalas micro y macroscópico. Esto crea oportunidades y desafíos para muchos campos, ya que abre la puerta a la producción de materiales, estructuras, sistemas y dispositivos con un enorme potencial práctico en energía, biomedicina, tecnologías ambientales, industria aeroespacial, tecnologías de la información y de la comunicación, microelectrónica e investigación militar, etcétera. $Y$ esto hace que se planteen al respecto retos políticos, sociales, económicos, éticos, cívicos y participativos.

Ante tal panorama, y como sucede con muchos campos científico-tecnológicos emergentes en sus primeros estadios, es frecuente que se disparen las expectativas de cambio social y se las asocie a algún tipo de revolución productiva (National Science and Technology Council, 2000:1) o a la solución de problemas como el cáncer, la contaminación o la falta de recursos energéticos. Pero todo ese potencial también es fuente de temores y preocupaciones entre la ciudadanía ante sus posibles riesgos.

En el ámbito internacional, la nanotecnología ha atraído la atención y el interés de los investigadores en comunicación y comprensión social de la ciencia de manera muy prematura. Hasta tal punto que puede considerarse un caso de estudio llamativo, ya que los investigadores se han dedicado a sondear a la opinión pública mucho antes de que ésta tuviera no ya una imagen formada sobre la nanociencia y la nanotecnología, sino una mínima familiaridad con ambas. La razón de este hecho se atribuye a lo ocurrido previamente con la biotecnología, especialmente con los alimentos manipulados genéticamente (y en general con los organismos genéticamente manipulados, conocidos en nuestro idioma por las siglas OGM), como señalan diversos autores (RS/RSE, 2004:59; Einsidiel y Goldenberg, 2004:28; Mehta, 2004:35; Priest, 2012:47). Esto llevó a posponer en gran medida las cuestiones y actividades concernientes a la comunicación y divulgación, es decir, las relacionadas con el fomento de una cultura básica sobre nanotecnología. De hecho los estudios e informes relacionados con la comunicación de la nanotecnología y la comprensión del conocimiento al respecto son más tardíos y creemos que escasos en relación con los de percepción pública (Crone y Koch, 2006; Castellini, Walejko, Holladay, Theim, Zenner y Crone, 2007; Hansen, Walhout, y van Est, 2008; Bonazzi, 2010; Hochgerner et al., 2010; OECD, 2012, Nanobiorase, s/f; Serena y Tutor, 2011; Gómez Ferri, 2012b). 
Anticipando lo que luego veremos más en detalle, en lo que respecta a los resultados de los estudios realizados, estos revelan un gran desconocimiento general de la nanotecnología por parte de la opinión pública, acompañado de una actitud más positiva que negativa. Eso supone una ruptura con la tendencia detectada ante campos científico-tecnológicos precedentes como el nuclear o el biotecnológico, donde ha predominado una visión amplificada del riesgo. Así, para la nanotecnología lo que de momento predomina es una "percepción atenuada del riesgo" (Priest, 2012:47). En lo que respecta a la cobertura mediática recibida por la nanotecnología en la prensa escrita, los primeros análisis, para Estados Unidos, mostraban que la atención recibida es escasa, que los temas más tratados son los de salud y medioambiente y, en todo caso, que en las informaciones predominan los tonos positivos y optimistas (Lewenstein, Gorss y Radin, 2005). La mayoría de los análisis realizados en diversos países confirman esta tendencia (Petersen, Anderson, Allan y Wilkinson, 2009).

\subsection{Las dificultades de comunicar la nanotecnología}

Como todo campo científico, la nanotecnología presenta una serie de dificultades de comprensión para el ciudadano que son comunes a la ciencia, como, por ejemplo, la complejidad, especialización y abstracción del conocimiento, la diversidad de contextos y audiencias, o la elección de los medios más adecuados. De hecho no es posible ni conveniente separar los problemas de divulgación y comprensión de la nanociencia y la nanotecnología del conjunto de la ciencia y la tecnología. No obstante, además de estas dificultades de orden general, habría una serie de aspectos más específicos a tener en cuenta. Sin ánimo de exhaustividad, hemos detectado las siguientes seis dificultades más específicas ${ }^{3}$ :

- De entrada, lo más común es señalar el obstáculo que supone comprender la escala a la que opera la nanotecnología -la mil millonésima parte del metro-, una escala muy alejada del mundo en el que operan los sentidos humanos. El trabajo, la investigación y los objetos nanoscópicos son poco intuitivos y, además, muy difíciles de imaginar y visualizar. El público en general, incluidos los escolares, tienen dificultades para diferenciar y situarse a esas escalas y para organizar e identificar entidades (Waldron, Sheppard, Batt y Spencer, 2005; Castellini, Walejko, Holladay, Theim, Zenner y Crone, 2007; Sánchez-Mora y Tagüeña, 2011).

- En relación con lo anterior, está el hecho de que para entender gran parte de lo que ocurre en la na- noescala se han de comprender procesos que ocurren a nivel cuántico, lo cual no es una cuestión asequible a la mayoría de público (Serena, 2013a:2).

- En tercer lugar, nos encontramos con la cuestión coyuntural y lógica de la novedad. Al ser la nanotecnología un campo emergente y puntero que está creando contemporáneamente nuevos conocimientos, técnicas y productos, resulta comprensible la existencia de un desconocimiento general.

- En cuarto lugar, hay que añadir la incertidumbre sobre el desarrollo futuro de la nanotecnología y del comportamiento de las nanopartículas y nanoestructuras. Estamos hablando de la probabilidad de riesgos y de beneficios, en los cuales están involucrados aspectos sociales, medioambientales y éticos para los que resulta muy difícil realizar predicciones más o menos acertadas debido al carácter emergente del campo (García Hom y Moles Plaza, 2013).

- En quinto lugar, surge la amplitud o extensión del campo abordado. No se trata de una ciencia o una tecnología en singular, sino más bien de un espacio multidisciplinar convergente, lo que implica procesos, conceptos, jergas, objetos, materiales, estructuras y productos de diferentes ramas de la ciencia y la ingeniería.

- Finalmente, aunque de menor importancia, pero relacionado con lo anterior, tampoco facilita las cosas la ocasional controversia sobre la verdadera entidad o sustantividad del campo de la nanotecnología-nanociencia. Hay investigadores que consideran la nanotecnología o la nanociencia como campos nuevos, con suficiente entidad y unidad, a veces distinguiendo entre una y la otra; a veces, no. Otros, en cambio, ponen en cuestión su existencia. Para estos no serían más que etiquetas publicitarias destinadas a atraer atención pública, mediática y financiera. Lo que existiría realmente son las disciplinas de siempre (física, química, ingeniería, etc.), sólo que centradas en la nanoescala.

\subsection{Problemática para la comprensión y la comuni- cación de la nanotecnología en España}

Junto a las dificultades de carácter más específico, la comprensión y la comunicación de la nanotecnología, podemos encontrar otra serie de dificultades que son de carácter contextual. Algunas de ellas son comunes y generales; otras son más bien propias de la sociedad española. A continuación se presentan sistematizadas cinco de ellas: 


\subsubsection{La ausencia de estudios sobre comprensión y comunicación pública de la nanotecnología}

En el ámbito internacional, las primeras encuestas sobre percepción pública contienen datos de 2001. Para la Unión Europea está el Eurobarómetro 55.2 Europeans, Science and Technology. Eurobarometer Special Survey 154 (Comisión Europea, 2001) y para Estados Unidos el estudio de Bainbridge (2002). A partir de ese año los estudios que amplían datos sobre los conocimientos, intereses y actitudes públicas ante la nanotecnología y sus posibles riesgos comienzan a ser cada vez más numerosos. Sin embargo, en el caso de España, destaca la ausencia de trabajos de investigación específicos o nacionales sobre comprensión y comunicación pública de la nanotecnología. Para saber acerca de la percepción pública de la nanotecnología únicamente contamos con los datos de los eurobarómetros globales de la Comisión Europea de los años 2001, 2002, 2005 y 2010, que no se centran en la nanotecnología, pero sí que incluyen preguntas al respecto.

Aparte de la escasa información que nos proporcionan los eurobarómetros, sólo muy recientemente se han publicado un par de trabajos sobre el tema. Sobre divulgación, está el artículo panorámico de Pedro Serena y Joaquín Tutor (2011), donde evalúan y hacen balance de la divulgación sobre nanociencia y nanotecnología en España, acompañado de una serie de recomendaciones para su mejora, dada su escasez. El segundo de ellos, de Giuseppe Veltri (2013), se centra en la imagen de la nanotecnología en la prensa española. En este trabajo, dicho autor efectúa un análisis semántico de la representación de la nanotecnología en la prensa española entre 1997 y 2009, tomando más de 600 noticias de los principales diarios. Veltri confirma el tono optimista que se encuentra en casi todos los análisis de prensa, el cual, además, se ha ido acentuando con el tiempo, ya que en los últimos años han desaparecido los aspectos más controvertidos, que sí estuvieron presentes en los primeros momentos. Invernizzi (2008), Novo y Borges (2010) y Körbes e Invernizzi (2010) han constatado esta tendencia en Brasil en la divulgación y en la publicidad tanto en general como en concreto sobre algunos productos de consumo.

\subsubsection{El desconocimiento sobre la nanotecnología}

El dato más destacado de los estudios de percepción pública realizados a nivel internacional, y en el que todos ellos coinciden, es el gran desconocimiento y falta de familiaridad que la gente tiene sobre nanotecnología (Comisión Europea, 2001:25; Bainbrid- ge, 2001:569; Waldron, Spencer y Batt, 2006: 571; Satterfield, Kandlikar, Beaudrie, Conti y Harthorn, 2009:753; Simons, Zimmer, Vierboom, Härlen, Hertel y Böl, 2009:1556). Esto puede ser lógico al principio. Sin embargo, y contra lo esperado, en la mayoría de países se observa un estancamiento en el nivel de familiaridad, con excepciones como Alemania y Australia (Simons, Zimmer, Vierboom, Härlen, Hertel y Böl, 2009:1558-9).

Con los datos que tenemos, en España, la familiaridad con la nanotecnología es de las más bajas de Europa, existiendo un gran segmento de la población española cuyo desconocimiento es absoluto (Comisión Europea, 2010a:34). En 2010 era del 67\%, porcentaje que, en nivel de familiaridad, situaba a la sociedad española en la posición veintidós de entre un total de veintisiete países. Es decir que siete de cada diez españoles no sabe de qué le están hablando cuando le hablan de nanociencia y nanotecnología. Y de los tres que sí han oído hablar de ellas, solo uno sabe de qué trata. Estos datos son consistentes con lo que apuntan estudios más amplios, en los que se pone de manifiesto el bajo nivel de cultura científica de la sociedad española, como es el caso del recientemente llevado a cabo por la Fundación BBVA, en el que se comparan diez países europeos, más los Estados Unidos (Bauer y Howard, 2013). Finalmente, los resultados también señalaban que el grado de interés que suscitaba la nanotecnología entre los españoles era escaso, en comparación con otros países y con respecto a otros ámbitos científicos y tecnológicos (Comisión Europea, 2001:37).

\subsubsection{La escasez de actividades de comunicación y de divulgación}

En España las actividades y publicaciones de divulgación sobre nanotecnología han sido escasas y han estado poco coordinadas, como también destaca la ausencia de tales contenidos en los diferentes niveles educativos, desde la primaria a la superior, pasando por la secundaria (Serena y Tutor, 2011:50; Serena, 2013b). Esto contrasta con la situación de otros países como Estados Unidos, Japón, Taiwán, Alemania o Francia, entre otros, donde se han elaborado planes específicos, que se han traducido en diferentes campañas de educación y divulgación, muchas de ellas dirigidas el público más joven, como nanoyou, nanodialogue, nanologue, nanocap, macospol, nanoplat, framingnano, nanotruck, nanocamp, nanoreisen, nanoboy (Comisión Europea, 2010b:15; Bonazzi, 2010:131). Aunque algunos de ellos son programas de la Comisión Europea, su alcance ha sido muy limitado 
y puntual en España, como también parece haberlo sido para el caso de las recomendaciones educativas y didácticas contenidas en programas más generales de desarrollo de la nanociencia y la nanotecnología.

\subsubsection{Falta de formación en comunicación pública de la ciencia}

Aunque, con la reciente institucionalización de las actividades de fomento y promoción de la cultura científica (Gómez-Ferri, 2012a), se ha incrementado la implicación de los científicos en las actividades de comunicación pública de la ciencia y se está reduciendo el tradicional divorcio entre científicos, divulgadores y periodistas científicos (Gonzalez-Alcaide, Valderrama-Zurián y Aleixandre-Benavent, 2009), lo cierto es que las instituciones en donde trabajan, así como las actividades comunicativas de cada uno de estos agentes responden a intereses y lógicas diferentes. Esto conlleva una traba para la divulgación de la ciencia (León, 1999). Por otro lado, la tendencia en aumento de que los científicos adopten el papel de comunicadores directos con la sociedad les supone una sobrecarga en demandas y exigencias sobre las funciones que tradicionalmente tenían asignadas. De algún modo se espera que los científicos, además de buenos investigadores, también deban ser buenos comunicadores, educadores y divulgadores.

En general, y por más que las demandas institucionales moralmente les demanden tal compromiso, los investigadores españoles, y los de la nanociencia y la nanotecnología no son una excepción, además de contar con pocos incentivos para comunicar sobre los campos que investigan, en general no han recibido una formación específica en comunicación pública de la ciencia. Es una tarea que realizan las más de las veces desde el compromiso y la satisfacción personal, el amateurismo y la voluntariedad (Torres, FernándezEsquinas, Rey-Rocha y Martín-Sempere, 2011:23; Serena y Tutor, 2011:50). La idea de que las universidades y centros de investigación deben satisfacer, además de las funciones de enseñanza y producción de saber, las de transferencia de conocimiento ha llevado, desde hace poco más de una década, a que se institucionalicen las actividades de divulgación científica (Gómez Ferri, 2012a). En la línea de incentivar y facilitar que los investigadores realicen tareas de divulgación científica están, en el ámbito europeo, algunos programas de la Comisión Europea y, en ámbito español, los promovidos por parte de fundaciones como la FECYT o COTEC, por no mencionar las de los propios centros universitarios y de investigación.
La presencia de la ciencia en los medios de comunicación más masivos, es decir, televisión, radio y prensa es deficitaria (Moreno-Castro, 2009:37). Sin embargo, los propios científicos siguen valorando la comunicación por tales medios como la más idónea, a pesar de tener una dinámica de funcionamiento ajena a los científicos y a la ciencia. Lo que valoran aquí es su alcance y su influencia. La presencia de los nuevos medios es cada vez mayor y sus posibilidades y accesibilidad son notorias, tanto para informar como para informarse. En relación con esto, la última encuesta de percepción pública de la ciencia realizada por la FECYT en el año 2012, muestra que Internet es ya la primera fuente de información sobre ciencia para los españoles (FECYT, 2012:15), lo cual se confirma en otros países (Brossard y Scheufele 2013:40). En este sentido, Corley y Scheufele (2010:22) constatan el efecto positivo que tienen los nuevos medios para los públicos menos informados, que están sirviendo para reducir la polarización informativa entre los públicos con mayores y con menores niveles culturales y educativos. En el siglo XXI se ha de apostar por los nuevos medios de comunicación, especialmente aquellos en los que el público pueda ser partícipe y no sea solo un sujeto pasivo ante la información.

\subsubsection{Un vacío en la participación y de los aspectos sociales, éticos, legales y medioambientales}

El declive teórico del denominado "modelo de déficit" (Cortassa, 2010:68) no se ha traducido en España en la adopción de alternativas de implicación participativa del público en las tareas de comunicación. Es verdad que, observando las actividades de divulgación de la ciencia que se realizan, se ha roto con el modelo tradicional de comunicación, en el sentido de que se han adoptado estrategias más lúdicas e interactivas que van desde carnavales a festivales, pasando por bares de la ciencia. Pero ese cambio se ha quedado corto a la hora de involucrar al público en procesos dialógicos y deliberativos, así como cuando se trata de integrar las implicaciones sociales de la ciencia y la tecnología en la comunicación. Si bien en el caso de la nanotecnología también son carencias que se denuncian en otros países (Kurath y Gisler, 2009: 559), involucrar al público upstream en los procesos de comunicación de la ciencia es una práctica inexistente en España (Revuelta, 2010). La nanotecnología brinda esa oportunidad de generar un diálogo más amplio entre ciencia y sociedad.

En suma, la novedad y heterogeneidad de la nanotecnología son dos condicionantes que pueden incidir en la falta de elección y estructuración de su conteni- 
do para comunicarlo al público, o mejor los públicos, dado que aquel no es un colectivo homogéneo. Establecer una serie de contenidos básicos y de dificultades para posteriormente definir los medios y las estrategias no es la única tarea a realizar, pero sí parece necesaria. Los expertos pueden paliar la situación de incertidumbre producida por la falta de información cuando un conocimiento no está estructurado y disponible en un momento dado. Las herramientas de prospectiva social, que se benefician del parecer de un conjunto de expertos, entre las que se encuentra el método Delphi, son adecuadas para ese propósito, ya que, además de la vertiente prospectiva, poseen asimismo una función orientadora. Para este cometido, los propios investigadores del campo de la nanotecnología pueden jugar un papel fundamental aportando sus conocimientos, juicios y estimaciones. Por esas razones, elegimos el método Delphi para los objetivos de esta investigación.

\subsection{Objetivos de la investigación}

De acuerdo con el planteamiento desarrollado hasta aquí, la investigación realizada pretendía identificar aquellos aspectos que desde el punto de vista de los expertos en nanotecnología facilitasen su comprensión a la opinión pública, así como los aspectos críticos de su comunicación. De manera más específica, la investigación se planteaba identificar los contenidos mínimos básicos de conocimiento sobre nanotecnología, establecer distintos niveles de dificultad de éste $y$, por último, detectar términos y conceptos que pueden ser fácilmente malinterpretados o malentendidos. Todo ello tras identificar las principales carencias al respecto existentes en la opinión pública española.

\section{METODOLOGÍA}

\subsection{Técnica de investigación}

El método Delphi se suele englobar dentro de los estudios de futuro o de prospectiva. $Y$ dentro de ellos, en las llamadas "técnicas subjetivas de previsión" (Landeta, 1999:15). Pero ese no es el único tipo de situación en que ha sido aplicada en el campo de la investigación. Diseñada por Helmer y Dalkey para la RAND Corporation en 1951 (Dalkey y Helmer, 1963:458), a partir de los trabajos previos de Kaplan, también es usada a menudo para determinar la diversidad de itinerarios que puede adoptar un fenómeno social y, de entre ellos, señalar los caminos que más probablemente desarrollará o adoptará da- das unas determinadas condiciones o escenarios. Es, por tanto, una técnica de investigación idónea para aproximarse a situaciones en las que deben tomarse decisiones, pero que se encuentran poco estructuradas, en las que predomina la incertidumbre o en las que los individuos generan definiciones de la situación con información nula, escasa o deformada. En tales situaciones, los expertos pueden desempeñar un papel fundamental, aportando sus conocimientos, juicios y valoraciones. Junto a la vertiente prospectiva, el método Delphi facilita la evaluación cualificada, lo que hace de dicho método una herramienta de extraordinaria utilidad en el campo de la investigación sobre la opinión pública y la comunicación aplicada. En términos operativos, el método Delphi pretende obtener "el consenso de opinión más fidedigno de un grupo de expertos" (Dalkey y Helmer, 1963:458). Consiste en "un programa cuidadosamente elaborado, que sigue una secuencia de interrogaciones individuales a través de cuestionarios, de los cuales se obtiene la información que constituirá la retroalimentación para los cuestionarios siguientes" (Helmer y Rescher, 1959:47).

La situación de la nanotecnología en España es semejante a la que acaba de describirse. Es decir, la información sobre lo que compete a la estructura de las nanocosas y sus interrelaciones con los humanos, el resto de seres vivos y el medio ambiente requiere una estructuración que facilite los procesos de comunicación y, por tanto, su comprensión pública. Esa es, pues, la razón por la que se consideró el método Delphi como la técnica más idónea para abordar los objetivos propuestos. Debe señalarse que la investigación realizada es, por un lado, una de las primeras en el área de comprensión de la nanotecnología en la opinión pública española y, por otro, la primera investigación sobre comprensión de la ciencia desarrollada a través de la técnica Delphi a nivel internacional, al menos hasta donde han podido constatar los autores de este trabajo.

\subsection{Elaboración de los cuestionarios}

Junto al panel de expertos, la aplicación de la técnica Delphi suele contar con un grupo coordinador que suele oscilar entre tres y cinco personas (Landeta, 1999:53). Las funciones de dicho grupo fueron establecer criterios para la elección de expertos, preparar o supervisar los cuestionarios e interpretar de los resultados. Este grupo estuvo integrado por los autores del artículo y tres investigadores del campo de la nanotecnología. 
Tras la formulación del proyecto, se elaboró un cuestionario que fue revisado y valorado por el grupo coordinador. Para evaluar este primer cuestionario, se realizaron nueve entrevistas semi-estructuradas a investigadores en nanociencia y nanotecnología de diferentes campos. Cinco de ellas se realizaron cara-a-cara y el resto telefónicamente entre el 14 de diciembre de 2011 y el 18 de enero de 2012. El propósito de las entrevistas fue comprobar la viabilidad del cuestionario y su adecuación al proceso de investigación. Los entrevistados fueron contactados al azar y, en un par de casos, a través del procedimiento bola de nieve sobre la base de la muestra que se describe en el subapartado siguiente. Al igual que posteriormente con los miembros del panel, en todo momento se garantizó el anonimato y confidencialidad de las respuestas.

Tras el análisis de las entrevistas, se confeccionó el cuestionario definitivo para la primera ronda, el cual contenía quince preguntas, ocho de respuesta abierta y siete de respuesta cerrada, dando la opción de introducir comentarios tanto en cada una de ellas como al final. Este cuestionario estaba dividido en cuatro bloques. El primero recogía datos e información de los participantes. El segundo incluía cuestiones relativas al conocimiento, comprensión y comunicación pública sobre nanociencia-nanotecnología. El tercero se ocupaba de escenarios futuros, riesgos y beneficios de la nanociencia-nanotecnología. El cuarto trataban temas relacionados con cuestiones de regulación y seguridad. En cuanto al cuestionario de la segunda ronda, se confeccionó a partir de las respuestas de la primera ronda y constó de ocho preguntas. Las tres primeras sobre comprensión y comunicación; la cuarta y la quinta sobre riesgos y beneficios, y las tres últimas sobre regulación y participación ciudadana.

\subsection{Procedimiento de recogida de la información}

El estudio se llevó a cabo en dos rondas que se realizaron entre los meses de marzo y junio de 2012. Aunque la aplicación tradicional de la técnica Delphi suele realizarse mediante correo postal, en la presente investigación el contacto con los investigadores se realizó por correo electrónico, por su mayor rapidez y menor coste. Se seleccionó una muestra de 196 investigadores activos en el campo de la nanotecnología y la nanociencia de una población total estimada de 4.991. Se fijó como objetivo de participación para la ronda final una cifra aproximada de treinta expertos, en consonancia con el criterio de la mayor parte de expertos que aconseja una participación final de 20 a 50 personas. Se estimó, por otro lado, una tasa de aceptación de entre el veinte y el treinta por ciento. De acuerdo con estas asunciones se consideró conveniente contar con una muestra inicial (expertos invitados) de 150 expertos, que se elevó hasta 196 para extremar las garantías finales de cobertura muestral.

Se seleccionó aleatoriamente una muestra de 196 posibles participantes a los que se les remitió un correo electrónico en el que se les explicaban los pormenores de la investigación y la demanda que se les formulaba, así como un adjunto consistente en una carta oficial en la que se les explicaba de manera detallada los objetivos de la investigación, su metodología y las características de la colaboración. Se les garantizó que la participación en el proceso sería obsequiada con el envío posterior del informe de resultados de la investigación. A los 60 que respondieron positivamente a la invitación, se les envió el cuestionario de la primera ronda, que fue cumplimentado por 48 expertos. A partir de las respuestas obtenidas se elaboró un segundo cuestionario que finalmente fue contestado por 38 expertos.

Tabla I. Participantes en el estudio delphi

\begin{tabular}{|l|c|c|}
\hline Participantes & $\mathbf{n}$ & Tasa de respuesta \\
\hline Número estimado de expertos existente en España & 4991 & -- \\
\hline Numero de expertos invitados a participar & 196 & $30,6 \%$ \\
\hline Respuestas positivas a la invitación & 60 & $24,5 \%$ \\
\hline Participantes en el cuestionario de la 1ạ ronda & 48 & $19,4 \%$ \\
\hline Participantes en el cuestionario de la 2a ronda & 38 & 28 \\
\hline
\end{tabular}




\subsection{Diseño de la muestra}

Una de las tareas que hubo que llevar a cabo para realizar el estudio es la confección del universo de expertos en nanotecnología. Se elaboró a partir de las bases de datos de tres redes de científicos. La principal de ellas fue la de la Red Española de Nanotecnología ${ }^{4}$, que contiene el listado más amplio de grupos de investigación en nanociencia y nanotecnología de España. El número de investigadores presentes en tal red hubiera sido suficiente para la elaboración de la muestra inicial del estudio Delphi, tanto por su tamaño como por el hecho de que su composición es muy heterogénea. Sin embargo, teniendo en cuenta que esta fue constituida principalmente por físicos y químicos se pensó que podría contener una sobrerrepresentación de tales disciplinas y fue completada con grupos de investigación de otras dos redes: la Plataforma española de Nanomedicina ${ }^{5}$ y el Centro de Investigación Biomédica en Red en Bioingeniería, Biomateriales y Nanomedicina (CIBER-BBN) ${ }^{6}$.

El listado final que se confeccionó no fue nominal, sino que en él se detallaba el número de componentes de cada uno de los grupos (centros o departamentos, en algunos casos). Dicho listado fue confeccionado entre finales de enero y principios de febrero de 2012. El número total resultante fue de 4.991 individuos; 4.565 de centros de investigación públicos o mixtos y 426 de empresas, los cua- les representan un $8,5 \%$ del total. Estos porcentajes se tuvieron en cuenta en el diseño de la muestra inicial (invitación) que reprodujo esas proporciones. Ello incluía personal técnico e investigadores predoctorales. Al final se excluyó a investigadores en nanociencia y nanotecnología en su periodo de formación predoctoral o que fuera personal técnico. Se consideró que por su nivel de conocimiento especializado los investigadores por encima del nivel predoctoral podrían ser expertos para valorar los conocimientos básicos y los riesgos que comunicar y transmitir al público.

Aunque el Delphi no persigue la representatividad estadística, se buscó el máximo grado de homología con el universo. Para ello se adoptó una estrategia de estratificación un tanto flexible, pues interesaba una cierta diversidad tipológica que incrementara la riqueza de las respuestas. Para ello se tuvo en cuenta la titularidad del centro de investigación (público, mixto, privado); el sexo del investigador y la categoría académica o profesional.

\subsection{Características del panel de expertos}

La tabla II contiene los datos de descripción de la muestra en relación al centro de investigación, sexo y categoría académica del investigador. Los perfiles obtenidos resultan acordes con la estratificación de estas variables en el universo de referencia.

Tabla II. Distribución final de los participantes según variables

\begin{tabular}{|l|l|c|c|}
\hline \multirow{5}{*}{ Tipo de centro de investigación } & & $\mathbf{n}$ & \% \\
\hline \multirow{5}{*}{ Sexo del investigador } & & 33 & 87,0 \\
\hline & Centros públicos & 3 & 3,0 \\
\cline { 2 - 4 } & Centros tecnológicos & 2 & 5,0 \\
\cline { 2 - 4 } & Empresas & 26 & 68,0 \\
\hline \multirow{5}{*}{ Categoría académica del investigador } & Hombres & 12 & 32,0 \\
\cline { 2 - 4 } & Mujeres & 10 & 26,3 \\
\hline & Titular de Universidad & 5 & 13,1 \\
\cline { 2 - 4 } & Profesor de Investigación & 4 & 15,8 \\
\cline { 2 - 4 } & Catedrático de Universidad & 2 & 10,5 \\
\cline { 2 - 4 } & Investigador Postdoctoral & 2 & 5,3 \\
\cline { 2 - 4 } & Investigador Científico & 1 & 5,3 \\
\cline { 2 - 4 } & Responsables de Comunicación & 8 & 2,6 \\
\cline { 2 - 4 } & Catedrático de Escuela Universitaria & 21,1 \\
\cline { 2 - 4 } & Otros & 2 & 2 \\
\hline
\end{tabular}




\section{RESULTADOS}

El desconocimiento existente en la sociedad española sobre nanociencia y nanotecnología nos ha llevado, en primer lugar, a determinar una serie de contenidos fundamentales de la nanotecnología que permitieran obtener una imagen suficientemente precisa de ésta. Con tal fin preguntamos a los expertos cuáles considerarían que son las cuestiones más básicas que la gente debería saber sobre ese nuevo campo. Para facilitar la respuesta, en la primera ronda, dábamos a los expertos la opción de señalar de uno a cinco ítems. Obtuvimos en total 155 ítems de respuesta, los cuales, tras ser categorizados quedaron resumidos en 26. A partir de este listado unitario, dado lo trabajoso que pudiera resultar que ordenaran los ítems, les pedimos a los expertos que valoraran cada uno de ellos de uno a tres, según los consideraran más o menos básicos. En la tabla III, ofrecemos los diez ítems que más puntuación obtuvieron en la segunda ronda, ordenados a partir de las medias resultantes para cada uno de ellos.

Estos diez ítems suponen el $55 \%$ de los veintiséis ítems categorizados. Los ítems que ocupan los primeros lugares $(10,2$, 3ㅇ, 6ㅇ) tienen que ver con aplicaciones y repercusiones positivas de la nanociencia y la nanotecnología, frente a los aspectos concernientes a los riesgos (9ㅇ), situados en una posición ulterior ${ }^{7}$. Asimismo, es de destacar que esos aspectos prácticos y vinculados a la cotidianeidad están por delante de los más estrictamente conceptuales (4우, 5ㅇ y 10ㅇ). Un caso significativo es el que tiene que ver con la cuestión del tamaño o escala (4ㅇ), que en la primera ronda era la respuesta de mayor frecuencia (1으), pasando a la cuarta en la segunda ronda. Entendemos que el cambio se debe a que, aunque en abstracto y conceptualmente se considera prioritaria, en el contexto de la divulgación científica priman aspectos que pueden servir para captar y atraer la atención de los públicos.

Los dos cambios de posición más significativos son los que competen a la justificación de la inversión económica en nanotecnología (8ㅇ), que en la primera ronda sólo recibió una mención. Al revés ocurre con el ítem 'la relación que hay entre la dimensión o escala y las diferentes propiedades que presentan los materiales o sistemas por los efectos clásicos y cuánticos del tamaño'. En la primera ronda era el segundo, con un $12 \%$ de mención, mientras que en la segunda ronda aparece en la posición número quince. Pensamos que esta cuestión pudo ser menos valorada al ser vista como de mayor dificultad que otras, aunque no somos ajenos al hecho de que pudiera haber influido la manera en que fue redactada al categorizarla.

Tabla III. Contenidos más básicos de la nanotecnología que el público debería conocer

\begin{tabular}{|c|c|c|}
\hline & 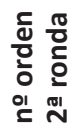 & 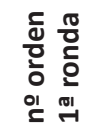 \\
\hline Usos y aplicaciones actuales de la nanociencia y la nanotecnología de interés para la sociedad & 1 & $3-4$ \\
\hline $\begin{array}{l}\text { Beneficios y ventajas desde el punto de vista científico, técnico, social, económico de la nanociencia y la } \\
\text { nanotecnología }\end{array}$ & 2 & $3-4$ \\
\hline Productos de uso cotidiano actualmente comercializados & 3 & 10 \\
\hline Las magnitudes o dimensiones a que se refiere la nanoescala & 4 & 1 \\
\hline El significado y caracterización de qué son la nanociencia y la nanotecnología & 5 & 5 \\
\hline Aplicaciones futuras de la nanociencia y la nanotecnología & 6 & $11-17$ \\
\hline La importancia y los objetivos de la nanociencia y la nanotecnología & 7 & 18 \\
\hline La razón de por qué invertir tantos recursos económicos en la nanociencia y la nanotecnología & 8 & $20-26$ \\
\hline Riesgos, costes y problemas de la nanociencia y la nanotecnología & 9 & $7-8$ \\
\hline $\begin{array}{l}\text { La identificación de diferentes entidades u objetos físicos, químicos y biológicos según sus tamaños } \\
\text { (átomos, moléculas, virus, células...) }\end{array}$ & 10 & 10 \\
\hline
\end{tabular}


Con respecto a los ítems que ocupan las posiciones que no aparecen en la tabla, de la once a la veintiséis, brevemente, señalar que estos conciernen a cuatro tipos de contenidos: (a) cuestiones básicas de la ciencia, (b) aspectos históricos y lingüísticos, (c) aclaratorios y (d) sobre seguridad. Ejemplos respectivos de cada uno de ellos son: 'la naturaleza atómica de la materia' (17으), 'los orígenes de la nanociencia y la nanotecnología' (23), que 'no todo lo que lleva la etiqueta "nano" lo es' (20), y 'cuestiones relativas a la seguridad de la nanomanipulación y de los nanomateriales (12ㅇ). Respecto de este último ítem echamos en falta un mayor énfasis en cuestiones sobre normativa, regulación y sobre los organismos y organizaciones encargadas de velar por su cumplimiento y por los intereses de los ciudadanos.

En conjunto, estos contenidos tienen una función orientativa. No se conciben con la exigencia de que el público deba saber todos ellos y todo sobre ellos. Por lo que se refiere a las acciones de divulgación o de información, el ordenamiento resultante no debe tomarse como algo cerrado y fijo, pero sí como una ordenación de carácter instrumental a tener presente a la hora de transmitir conocimientos al público o también de comprobar si están familiarizados con ellos. Por otro lado, a pesar de la amplitud de temas, cabe el hecho que algún interés o inquietud social no esté recogido en esta batería básica. Sobre esto ya hemos señalado la necesidad de completar esta información con la que se pueda obtener de los públicos y de otros expertos vinculados al campo procedentes de la comunicación y las ciencias sociales.

Junto a este primer aspecto, pensamos que, conceptualmente, el acercamiento de la nanotecnología al público tiene que tener en cuenta dos elementos fundamentales más: el nivel de dificultad de los conceptos y la posibilidad de que sean malinterpretados. Para lo primero se pidió a los expertos que indicaran aquellas cuestiones que, siendo básicas, juzgaban de más difícil comprensión. Para esta cuestión, el número de ítems de respuesta fue de noventa, que finalmente quedaron categorizados en veinte. En la segunda ronda, estos ítems fueron evaluados por los expertos de uno a tres, de acuerdo a su nivel de dificultad. En la tabla IV se recogen los diez ítems, según su media de puntuación. Tal listado no debe entenderse como un listado de conceptos complejos o de especial dificultad de la nanotecnología. Son conceptos más o menos fundamentales para la comprensión pública de la nanotecnología, pero que, comparativamente, pueden revestir mayores dificultades.

Tabla IV. Contenidos básicos sobre nanotecnología según su nivel de dificultad para el público

\begin{tabular}{|c|c|c|}
\hline & 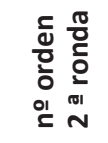 & 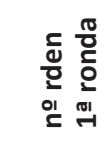 \\
\hline La spintrónica & 1 & $12-20$ \\
\hline El autoensamblado molecular & 2 & $12-20$ \\
\hline $\begin{array}{l}\text { Las razones de por qué en la nanoescala cambian las propiedades de ciertos materiales y se } \\
\text { comportan de manera distinta a como lo hacen a escalas mayores }\end{array}$ & 3 & 1 \\
\hline $\begin{array}{l}\text { Cómo se manipula la material a escala nanométrica y cómo se consigue que los materiales tengan } \\
\text { determinadas propiedades }\end{array}$ & 4 & $6-8$ \\
\hline La modulación de las propiedades de los nanomateriales & 5 & $12-20$ \\
\hline Los procesos artificiales y naturales de nanoestructuración & 6 & $12-20$ \\
\hline $\begin{array}{l}\text { Los equipamientos y el instrumental para estudiar y manipular en la nanoescala y su } \\
\text { funcionamiento }\end{array}$ & 7 & $9-11$ \\
\hline Las técnicas de control y caracterización de la materia a escala nanométrica & 8 & $4-5$ \\
\hline La funcionalización de nanomateriales & 9 & $12-20$ \\
\hline El propósito que tiene hacer componentes a escala nanométrica y cómo se hacen & 10 & $12-20$ \\
\hline
\end{tabular}


De estos diez ítems, en concreto el tercero, cuarto y sexto también fueron señalados por los expertos como conceptos básicos (tabla III). Estos resultados deben servir para reforzar el esfuerzo en su explicación, buscando estrategias o recursos que sirvan para captar el interés del público y faciliten su comprensión, más que para rehuir o evitar su empleo.

Desde un punto de vista analítico, la mayoría de las cuestiones señaladas (incluyendo las que no hemos incorporado al listado) tienen que ver con: (a) procesos y procedimientos de la nanotecnología. (Prácticamente las primeras de la tabla IV son de este tipo.); (b) nuevos materiales (12ㅇ), como es el caso del grafeno o los nanotubos; (c) cuestiones conceptuales como la escala o magnitud (16ㅇ) o (d) aspectos de índole contextual. A la hora de llevar a cabo las tareas de información y divulgación se habrá de tener presente lo que consideramos un doble grado de dificultad: procesos (a), por una parte, y el resto de cuestiones (b, $c, d)$, por la otra.

Por último, además de la falta de conocimientos y de la complejidad de las cuestiones implicadas, un tercer problema para la compresión pública de la nanotecnología es el que tiene que ver con la mala interpretación de algunos de sus conceptos o términos. Esta mala interpretación puede dar lugar al equívoco, como también producir confusión o preocupación. Con el fin de identificar algunos de los posibles conceptos o temas susceptibles de ser fácilmente malinterpretados o que se prestarán al equívoco, pedimos a los expertos en la primera ronda que señalaran, sin límite, aquéllos que les pareciera oportuno. El número total de ítems resultante (103) quedaron clasificados en cuarenta y siete. De ellos, treinta y cuatro con una única referencia y siete con dos. El resultado apunta a la gran cantidad de ítems que los expertos consideran fácilmente interpretables de manera errónea. El desconocimiento público y la amplitud y heterogeneidad del campo pueden ser dos razones explicativas de esto. Sin embargo, el objetivo era encontrar pautas más concretas de mala interpretación. En este sentido hallamos una básica en el hecho de que muchos de los conceptos que se emplean en nanotecnología son términos del lenguaje común, a los que se ha antepuesto el prefijo 'nano's. Esta circunstancia puede llevar al público no experto a pensar (erróneamente) en los objetos, dispositivos o estructuras de la nanoescala en función de los objetos cotidianos que le dan nombre.

Con respecto a los otros términos o cuestiones señaladas por los expertos, el primer lugar lo ocupan, en conjunto, los aspectos toxicológicos de los nanomateriales o conceptos como 'nanotocixidad' (10\%), bien por la dificultad de esas cuestiones o porque el mero hecho de mencionar el tema puede hacer pensar que todos los nanomateriales son peligrosos o tóxicos. En segundo lugar, están términos como 'nanorobots' o'nanobots' (9\%), seguramente por su vinculación con las fantasías catastrofistas del novelista Michael Crichton (2002) $)^{9}$. A continuación aparecen expresiones como 'nanopartícula' (7\%) o el término 'manipular' (3\%), porque en el imaginario social pueden vincularse a actividades que impliquen riesgo o peligro. Entre ambas, destacan los términos 'nanopunto' o quantum dot ('punto cuántico') (5\%), de gran peso y uso en este campo científico.

Sobre las posibilidades de interpretación equivocada, algunos expertos han indicado que no se trata de una cuestión de palabras -y que depende de la habilidad del comunicador para evitar la producción de equívocos-, o bien que cualquier término puede ser malinterpretado. No obstante, el hecho de poseer una mínima base conceptual sobre la nanotecnología, así como el énfasis a la hora de clarificar aquellos términos que se prestan más fácilmente a ser distorsionados por su asociación con otros, son prevenciones útiles a tener en cuenta cuando se informe sobre este campo científico y tecnológico.

\section{CONCLUSIONES}

En esta investigación partíamos del gran desconocimiento existente en la sociedad española sobre un campo científico y tecnológico emergente como es la nanotecnología, así como del contexto más general en el que hemos de situar la comprensión y comunicación de la ciencia. Esa situación de ignorancia, además de no ser coherente con la lógica de las sociedades democráticas, también podría ser contraproducente para la nanotecnología a medio o, incluso, a corto plazo. Ante todo esto, consideramos que la implicación y el concurso de los expertos era la mejor opción para seleccionar y organizar un conjunto de contenidos sobre nanotecnología destinados a la comunicación pública. Para ello realizamos una consulta a investigadores de dicho campo con el fin de que identificaran unos contenidos básicos, así como posibles dificultades de comprensión.

Los resultados proporcionan una información valiosa sobre cuáles son los contenidos de este nuevo campo científico y tecnológico que los investigadores consideran fundamentales para el conocimiento público. Son contenidos que científicos, periodistas y 
divulgadores pueden tener como referencia cuando divulguen o comuniquen. A la hora de organizarlos, comenzar por los aspectos prácticos y los posibles beneficios servirá probablemente para interesar al público (como sucede con otras investigaciones científicas y desarrollos tecnológicos), seguidamente de la cuestión de la escala y las dimensiones. En general, los contenidos concernientes a los procesos en la nanoescala deberán posponerse en favor de otros contenidos más básicos como el hecho de que la nanociencia nanotecnología no es ni tan exótica ni tan nueva como se la presenta. Finalmente, se ha de ser consciente de las interferencias semánticas que pueden provocar una serie de términos, provenientes de otros campos, sean científicos o cotidianos, cuando se emplean técnicamente en la nanotecnología, ya que pueden llevar al público no experto a pensar en los objetos, dispositivos o estructuras de la nanoescala en función de los objetos cotidianos cuyo nombre es adoptado.

Este estudio se planteó como un intento de contribuir a la mejora de la comunicación social de la nanotecnología. En este sentido es una contribución parcial, ya que necesitaría ser complementada con nuevas estrategias de elección de las formas, medios y mecanismos adecuados para comunicar la nanotecnología, así como el nivel de implicación pública a que se aspira; además de las demandas informativas de los ciudadanos, los cuales, como público, no son una entidad homogénea, y del punto de vista de comunicadores y expertos de las ciencias sociales. Pero eso era algo que no competía propiamente a la pericia de este grupo de expertos. Las cuestiones relacionadas con la selección y organización de contenidos y mensajes requieren más trabajo en esa línea, la cual necesita complementarse con la adecuada elección de medios y formatos, y la elaboración de los mensajes. Finalmente los actores implicados deben empezar a concebir su tarea como un trabajo que conlleva la coordinación de distintos profesionales como ingenieros, científicos sociales, educadores, periodistas o especialistas en nuevas tecnologías de la comunicación.

\section{AGRADECIMIENTOS}

Estamos agradecidos a Pedro Serena, Fernando Sapiña y Guillermo Muñoz Matutano por la ayuda que nos han prestado durante el proceso de investigación. También lo estamos con los evaluadores anónimos por sus valiosos comentarios y críticas al texto. Finalmente, indicar que la realización de este trabajo ha sido financiada parcialmente con la concesión del proyecto de investigación "Nanotecnología y conocimiento: un instrumento para fomentar la gobernanza social de la nanotecnología a través de un público atento", dentro del Programa de Apoyo a la Investigación de la Universidad de La Laguna para el año 2013.

\section{NOTAS}

1 La distinción entre nanociencia y nanotecnología no siempre resulta fácil de trazar. Por eso, y por abreviar, generalmente emplearemos la denominación "nanotecnología" refiriéndonos a ambas.

2 Ambos trabajos forman parte de un número monográfico de la revista Mundo Nano, donde se analiza el estado de la divulgación y la formación en nanociencia y nanotecnología en Argentina, Brasil, Colombia, Cuba, Chile, España, México, Perú, Portugal y Venezuela, países que forman parte de la Red $\mathrm{Na}$ nodyf, una red iberoamericana orientada a la divulgación de la nanociencia y la nanotecnología.
3 Es un dato que recoge la diversa literatura especializada en el tema, y algo que también hemos constatado en nuestra actividad investigadora.

4 http://www.nanospain.org

5 http://www.nanomedspain.net

6 http://www.ciber-bbn.es/

7 Por ejemplo, en la posición 12a se menciona las cuestiones de seguridad. Por motivos de espacio no hemos introducido aquí la tabla completa. Tanto la III como la IV se pueden consultar completas en el informe "Comprensión pública de la nanociencia y la nanotec- nología. Informe de los resultados de un estudio Delphi (https://www.academia. edu/4488418/Comprension publica de_la_nanociencia_y_la_nanotecnologia. Informe de los resultados de un_estudio_Delphi).

8 Ejemplos de ello son: nanopunto, nanotijeras, nanohilo, nanoaguja, nanopinza, nanoantena.

9 Este conocido autor imagina en su novela Prey una amenazante "plaga gris" de diminutos robots invisibles al ojo humano (nanobots), autoreplicándose descontroladamente. 
Bainbridge, W. S. (2002). "Public attitudes towards nanotechnology", Journal of Nanoparticle Research, vol. 4, no. 6, pp. 561-570. doi: http://dx.doi. org/10.1023/A:1022805516652

Bauer, M. W. y Howard, S. (2013). The Culture of Science in Modern Spain: An Analysis of Public Attitudes Across Time, Age Cohorts and Regions. Madrid: Fundación BBVA.

Bodmer, W. et al., (1985). Public Understanding of Science. London: Royal Society.

Bonazzi, M. (2010). Communicating nanotechnology. Why, to whom, saying what and how? An action-packed roadmap towards a brand new dialogue. Bruselas: Directorate-General for Research. Recuperado de http://ftp.cordis.europa.eu/pub/nanotechnology/docs/communicating-nanotechnology_en.pdf

Brossard D. y Scheufele, D. A., "Science, New Media, and the Public", Science, no 339, 2013, p.40. doi: http://dx.doi. org/10.1126/science.1232329

Castellini, O. M.; Walejko, G. K.; Holladay, C. E.; Theim; T.. J.;. Zenner G. M. y Crone, W. C. (2007). “Nanotechnology and the public: Effectively communicating nanoscale science and engineering concepts", Journal of Nanoparticle Research, no. 9, pp. 183-189. doi: http:// dx.doi.org/10.1007/s11051-006-9160-z

Calvo Hernando, M. (1997). Manual de Periodismo Científico. Barcelona: Bosch.

Comisión Europea (2001). Eurobarometer 55.2: Europeans, Science and Technology. Eurobarometer Special Survey 154. Bruselas, Directorate General Press and Communication. Recuperado de http:// ec.europa.eu/research/press/2001/ pr0612en-report.pdf

Comisión Europea (2010a). Eurobarometer 73.1: Biotechnology. Special Eurobarometer 341. Bruselas: Directorate General Press and Communication. Recuperado de http://ec.europa. eu/public_opinion/archives/ebs/ ebs_341_en.pdf

Comisión Europea (2010b). Knowledge, Attitudes and Opinions on Nanotechnology across European Youth Analysis from a specific survey carried out in 25 EU countries. Bruselas: Office of the European Union. Recuperado de http:// ec.europa.eu/research/industrial_technologies/pdf/knowledge-attitudeopinions-on-nanotech_en.pdf
Corley, E. A., y Scheufele, D. A. (2010). "Outreach gone wrong? When we talk nano to the public, we are leaving behind key audiences", The Scientist, vol. 24, no1, p. 22

Cortassa, C. G. (2010). "Del déficit al diálogo, ¿y después? Una reconstrucción crítica de los estudios de comprensión pública de la ciencia", Revista CTS, vol. 5, no 15, pp. 117-124. Recuperado de http://revistacts.net/files/Volumen\%205\%20-\%20 N\%C3\%BAmero\%2015/Cortassa.pdf

Crone, W. C. y Koch, S. E. (eds.) (2006). Bringing nano to the public: A collaboration opportunity for researchers and museu$m s$. St.Paul: NISE Network. Recuperado de http://www.nisenet.org/catalog/ tools-guides/bringing-nano-public

Crichton, M. (2002). Prey. New York: Harper Collins.

Dalkey, N. C. y Helmer, O. (1963). “An Experimental Application of the Delphi method to the use of experts", Management Science, vol. 9, no. 3, pp. 458-467.

Einsiedel, E. F. y Goldenberg, L. (2004). "Dwarfing the social? Nanotechnology lessons from the biotechnology front", Bulletin of Science, Technology \& Society, vol. 24, no. 1, pp. 28-33. doi: http://dx.doi.org/ 10.1177/0270467604263110

FECYT (2012). VI Encuesta de Percepción Social de la Ciencia 2012, Madrid: FECYT. Recuperado de http://www.fecyt. es/fecyt/docs/tmp/363174605.pdf

García Hom, A. y Moles Plaza, R. J. (2013). "Gestionando entornos sociotécnicos complejos: la gobernanza del riesgo en las nanotecnologías", en Mundo Nano. Revista interdisciplinaria en Nanociencia y $\mathrm{Na}$ notecnología, vol. 6, no. 10, pp.: 86-100. Recuperado de http://www.mundonano. unam. $\mathrm{mx} / \mathrm{pdfs} / \mathrm{mundonano10.pdf}$

Gómez Ferri, J. (2012a). "Cultura: sus significados y diferentes modelos de cultura científica", en Revista Iberoamericana de Educación, no 58, pp. 15-33. Recuperado de http://www.rieoei.org/ rie58a01.pdf

Gómez Ferri, J. (2012b). “La comprensión pública de la nanotecnología en España", Revista Iberoamerica de Ciencia, Tecnología y Sociedad, vol 7 no 20: 177207. Recuperado de http://www.revistacts.net/files/Volumen\%207\%20-\%20 N\%C3\%BAmero\%2020/gomezferricORREGIDOFINAL.pdf
Gonzalez-Alcaide, G.; Valderrama-Zurián, J. C. y Aleixandre-Benavent, R. (2009). "La investigación sobre la divulgación de la ciencia en España: situación actual y retos para el futuro", Arbor, vol. 73, pp. 861-869. doi: 10.3989/ arbor.2009.738n1058

Gregory, J. y Miller, S. (1998). Science in Public. Cambridge: Basic Books.

Hanssen, L.; Walhout, B. y van Est, R. (2008). Ten lessons for a nanodialogue: The Dutch debat about nanotechnology thus far. The Hague: Rathenau Institute, TA-report 0802, Recuperado de http:// www.rathenau.nl/uploads/tx_tferathenau/Ten_lessons_for_a_nanodialogue_2008_01.pdf

Hayhurst, R., Heckl, W. M.; Maglio, G.; Türk, V. y Bennett, D. (2007). "Talking NanoWhat Makes Nanotechnology Special". En Claessens, M. (ed.). Communicating European Research (pp. 229-232). Dordrecht: Springer. doi: http://dx.doi. org/10.1007/1-4020-5358-4

Helmer, O. y Rescher, N. (1959). "On the epistemology of the inexact science", Management Science, vol. 6, no. 1, pp. 25-53.

Hochgerner, J. et al. (2010). NanoyouWP1. Report on the Analysis of Survey Responses. Recuperado de http:// nanoyou.eu/attachments/495_NANOYOU_D1.2_ZSI.pdf

Invernizzi, N. (2008). "Visões de futuro: nanociência e nanotecnologia no Jornal da Ciência", VII Jornadas Latinoamericanas de Estudios Sociales de la Ciencia y Tecnología: Rio de Janeiro, Brasil. Recuperado de http://www.necso.ufrj.br/ esocite2008/resumos/35807.htm

Körbes, C. y Invernizzi, N. (2010). "Tecnologia e a difusão da ideia de progresso da ciência", VIII Jornadas Latinoamericanas de Estudios Sociales de la Ciencia y Tecnología: Buenos Aires, Argentina. Recuperado de http://www. esocite2010.escyt.org

Kurath, M. y Gisler, P. (2009). “Informing, involving or engaging? Science communication, in the ages of atombio- and nanotechnology", Public Understanding of Science, vol. 18, n으, pp. 559-573. doi: http://dx.doi.org/ 10.1177/0963662509104723

Landeta, J. (1999). El método Delphi. Una técnica de previsión para la incertidumbre. Barcelona: Ariel. 
Lee, C. J. y Scheufele, D. A. (2006). "The influence of knowledge and deference toward scientific authority: A media effects model for public attitudes toward nanotechnology", Journalism \& Mass Communication Quarterly, vol. 83, no 4, pp. 819-834.

León, B. (1999). El documental de divulgación científica. Barcelona: Paidós.

Lewenstein, B. V.; Gorss, J. y Radin, J. (2005). "The Salience of Small: Nanotechnology Coverage in the American Press 1986-2004". International Communication Association, 26-30 May 2005. New York, Recuperado de https:// ecommons.library.cornell.edu/bitstream/1813/14275/2/LewensteinGorssRadin.2005.NanoMedia.ICA.pdf

Mehta, M. D. (2004). “From Biotechnology to Nanotechnology: What Can We Learn From Earlier Technologies?". Bulletin of Science, Technology \& Society, vol. 24, no 1,pp. 34-39. doi: http://dx.doi. org/10.1177/0270467604263119

Moreno-Castro, C. (2009). "Los medios, el público y la ciencia. Una relación que no progresa adecuadamente". En FECYT, Percepción social de la ciencia y la tecnología en España 2008, (pp.21-38). Madrid: FECYT.

Nanobiorase (s/f). Public Perceptions and Communication about Nanobiotechnology, NanoBio-RAISE. Delft: Co-ordination office,. Recuperado de http://files. nanobio-raise.org/Downloads/NanoPublicFINAL.pdf

National Science and Technology Council (2000). National nanotechnology initiative: Leading to the next industrial revolution. Washington, D.C.: Interagency Working Group on Nanoscience, Engineering and Technology. Disponile en http://www.whitehouse.gov/files/ documents/ostp/NSTC\%20Reports/ NNI2000.pdf

Novo, M. y Borges, E. L. (2010). "Nanotecnologia e as constituições de gênero", VIII Congresso Iberoamericano de Ciência, Tecnologia e Gênero: Curitiba, Brasil. Recuperado de http://files.dirppg. ct.utfpr.edu.br/ppgte/eventos/cictg/ conteudo_cd/E11_Nanotecnologia

OECD (2012). Planning guide for public engagement and outreach in nanote- chnology. OECD. Recuperado de http:// www.oecd.org/sti/biotechnologypolicies/49961768.pdf

OEI (2009). La nanotecnología en Iberoamérica. Situación actual y tendencias. Recuperado de http://www.oei.es/salactsi/nano.pdf

Petersen, A.; Anderson, A.; Allan, S. y Wilkinson, C. (2009). "Opening the black box: scientists' views on the role of the news media in the nanotechnology debate", Public Understanding of Science, vol. 18, n으, pp. 512-530. doi: http://dx.doi. org/10.1177/0963662507084202

Phillips, L. (2012). "Armed resistance", Nature, nㅇ 488, 30 Agosto, pp. 576-579.

Priest, S. H. (2012). Nanotechnology and the Public: Risk Perception and Risk Communication. London: CRC Press, Taylor \& Francis Group.

Revuelta, G. (2010). DG Research Monitoring Policy and Research Activities on Science in Society in Europe (MASIS) National Report, SPAIN. Recuperado de http://www.masis.eu/files/reports/ MASIS_SPAIN_report.pdf

Sánchez-Mora, M.C. y Tagüeña, J. (2011). "El manejo de las escalas como obstáculo epistemológico en la divulgación de la nanociencia", Mundo nano. Revista interdisciplinaria en Nanociencia y Nanotecnología, vol. 4, no 2, pp.83-102.

Satterfield, T.; Kandlikar, M.; Beaudrie, C.E.H.; Conti, J. y Harthorn, B. H. (2009). "Anticipating the perceived risk of nanotechnologies", Nature Nanotechnology, no. 4, pp. 752-758. doi: http://dx.doi. org/ 10.1038/NNANO.2009.265

Scheufele, D. A. (2006). “Nano doesn't have a marketing problem... yet", en Nanotoday, vol. 2, no 5, pp. 48 .

Serena, P. A. y Tutor, J. D. (2011). "La divulgación y la formación de la nanociencia y la nanotecnología en España: un largo camino por delante", Mundo nano. Revista interdisciplinaria en Nanociencia y Nanotecnología, vol. 4, no. 2, pp. 48-58. Recuperado de http://www.mundonano.unam.mx/pdfs/mundonano7.pdf

Serena, P. (2013a). "La Nanociencia y la Nanotecnología: en la frontera de lo pequeño", Revista Española de Física, vol. 27, n으 1. pp. 1-5.
Serena, P. (2013b). "Acercando la nanotecnología a la sociedad: la exposición 'un paseo por el nanomundo'", Revista Digital Universitaria, vol. 14, no 4. Recuperado de http://www.revista.unam.mx/ vol.14/num4/art29/index.html

Simons, J.; Zimmer, R.; Vierboom, C.; Härlen, I.; Hertel, R. y Böl, G. F. (2009). “The slings and arrows of communication on nanotechnology", Journal of Nanoparticle Research, no 11, 2009, pp. 15551571. doi: http://dx.doi.org/10.1007/ s11051-009-9653-7

Torres, C.; Fernández-Esquinas, M.; ReyRocha, J. y Martín-Sempere, M. J. (2011). "Dissemination practices in the Spanish research system: scientists trapped in a golden cage", Public Understanding of Science, vol. 20, no 1, pp. 12-25. doi: http://dx.doi. org/10.1177/0963662510382361

Veltri, G. A. (2013). "Viva la Nano-Revolución! A Semantic Analysis of the Spanish National Press", Science Communication, vol. 35 no. 2, pp. 143-167. doi: http://dx.doi. org/10.1177/1075547012440353

Tutor, J. y Serena, P. A. (2011), “Situación de la divulgación y la formación en nanociencia y nanotecnología en Iberoamérica", Mundo nano. Revista interdisciplinaria en Nanociencia y Nanotecnología, vol. 4, no 2. Recuperado de http://www. mundonano.unam. $\mathrm{mx} / \mathrm{pdfs} /$ mundonano7.pdf

Waldron, A.M.; Sheppard, K.; Batt, C. y Spencer, D. (2005). "Too small to see: Educating the next generation in nanoscale science and engineering". En C.S. Kumar, Hormes, J. y Leuschner, C. (eds.), Nanofabrication towards biomedical applications: Techniques, Tools, Applications, and Impact. (pp. 375-389). Weinheim: Wiley-VCH Verlag GmbH \& Co. doi: http://dx.doi. org/10.1002/3527603476.ch15

Waldron, A.; Spencer, D. y Batt, C. (2006). "The current state of public understanding of nanotechnology", Journal of Nanoparticle Research, vol. 8, no. 5, pp. 569-575. doi: 10.1007/s11051006-9112-7 